\title{
Molecular Identification of Sclerotinia trifoliorum and Sclerotinia sclerotiorum Isolates from the United States and Poland
}

Anna Baturo-Ciesniewska, Department of Phytopathology and Molecular Mycology, UTP University of Science and Technology, Kordeckiego 20, 85-225 Bydgoszcz, Poland; and Carol L. Groves, Department of Plant Pathology; Kenneth A. Albrecht, Department of Agronomy; and Craig R. Grau, David K. Willis, and Damon L. Smith, Department of Plant Pathology, University of Wisconsin-Madison, Madison 53706

\begin{abstract}
Symptoms of clover rot caused by Sclerotinia trifoliorum or S. sclerotiorum are identical, making differentiation and identification of the causal species difficult and time consuming. Polymerase chain reaction (PCR) amplification and nucleotide sequencing were used to examine 40 isolates of $S$. trifoliorum ( 29 from Poland, 11 from the United States) and 55 isolates of $S$. sclerotiorum (26 from Poland, 29 from the United States). We determined that amplification of the $\beta$-tubulin and calmodulin genes with TU1/TU2/TU3 and SscadF1/SscadR1 PCR primers and the presence of introns and single-nucleotide polymorphisms (SNP) within the ribosomal DNA (rDNA) as detected with NS1/NS8 and internal transcribed spacer (ITS)1/ITS4 PCR primers

are effective for rapidly and accurately differentiating between the two species of Sclerotinia. In addition, our research revealed a lack of intraspecies variation within S. sclerotiorum isolates from the United States and Poland using these same molecular markers. We detected a relatively high degree of intraspecies variability among isolates of $S$. trifoliorum from the United States and Poland using the presence of introns and SNP within the rDNA. SNP and nuclear small-subunit rDNA analyses revealed distinct groups of $S$. trifoliorum among the isolates used in this study. The results of this study provide useful information for clover breeders and pathologists looking to develop clover varieties with durable resistance.
\end{abstract}

Kura or Caucasian clover (Trifolium ambiguum M. Bieb.) is a rhizomatous, persistent legume (Bryant 1974; Speer and Allinson 1985; Taylor and Smith 1997) that tolerates frequent defoliation in monoculture (Peterson et al. 1994), is reported to be adapted to a wide range of soil types (Speer and Allinson 1985), and survives long, cold winters (Sheaffer and Marten 1991). It is native to the Caucasus Region (Bryant 1974; Kannenberg and Elliott 1962; Speer and Allinson 1985) and was introduced outside its center of origin in the first half of the twentieth century (Bryant 1974). It has provided exceptional animal performance in pasture (Mouriño et al. 2003) and as silage (Kammes et al. 2008) when grown in mixture with grasses because of extreme persistence and excellent nutritive value. Commercial use of Kura clover is concentrated in the northern United States, Canada, the South Island of New Zealand, and Australia (Patrick et al. 1994; Peterson et al. 1994; Pryor et al. 1996; Steiner and Snelling 1994; Taylor 2008; Zemenchik et al. 2000). Kura clover has been considered resistant to most virus, root, stem, and foliar pathogens and nematodes that seriously damage red and white clover (Barnett and Gibson 1975; Pederson and McLaughlin 1989; Pederson and Windham 1989). It has also been reported to be highly resistant to Sclerotinia trifoliorum Erikss. (Barnett and Diachun 1985, Taylor and Smith 1997), one of the most destructive pathogens of forage legume crops (Kohn 1979). These desirable characteristics have generated interest in using Kura clover as a forage legume in Poland. In 1997 and again in 2009, attempts were made to establish Kura clover from cultivars considered to be resistant to $S$. trifoliorum in the United States. These cultivars, grown in Poland, were nearly decimated due to disease (Baturo-Ciesniewska et al. 2013; Harasimowicz-Hermann and Andrzejewska 1999). When establishment was successful, most plants died within 2 years (Andrzejewska et al. 2016). The damage observed on Kura clover in Poland was attributed to clover rot caused by fungi in the genus Sclerotinia (Andrzejewska et al. 2014; Baturo-Ciesniewska et al. 2013).

Corresponding author: D. L. Smith; E-mail: damon.smith@wisc.edu

Accepted for publication 18 August 2016.

() 2017 The American Phytopathological Society
Clover rot is typically caused by $S$. trifoliorum and S. sclerotiorum (Lib.) de Bary (Kohn 1979; Vleugels et al. 2012). Symptoms caused by both species on clover are identical, making the differentiation and identification of species causing clover rot difficult (Pratt and Rowe 1995; Saharan and Mehta 2008; Vleugels et al. 2013). Identification of the two species has historically been based on morphology and biological or cultural characteristics. However, the only unequivocal morphological differentiation between the two species requires observation of ascospore morphology resulting from the induction of carpogenic germination of sclerotia in the laboratory. This process can be very tedious and time consuming, requiring up to several months to complete (Baturo-Ciesniewska et al. 2013; Kohn 1979; Marum et al. 1994; Njambere et al. 2008). Also, some isolates of S. trifoliorum are heterothallic and require a compatible mating strain in order to induce carpogenic germination and ascospore formation (Vleugels et al. 2012).

In an effort to increase the efficiency of Sclerotinia spp. identification, several different techniques have been applied to facilitate accurate identification: restriction fragment length polymorphisms (Kohn et al. 1988); differential growth rates on agar media (Tariq et al. 1985); sequence divergence within the ribosomal DNA (rDNA) internal transcribed spacer (ITS) (Carbone and Kohn, 1993); presence or absence of group I introns in the nuclear small-subunit (nSSU) of the rDNA (Powers et al. 2001); single-nucleotide polymorphisms (SNP) within the intergenic spacer, calmodulin, and ras protein gene differences (Andrew and Kohn 2009); SNP within the rDNA intergenic spacer region (Njambere et al. 2008); polymerase chain reaction (PCR) amplification of the $\beta$-tubulin gene (Vleugels et al. 2012); and discriminatory simplex and multiplex PCR (AbdElmagid et al. 2013). Some of these techniques produce reliable results but require specialized equipment and supplies and are time consuming to perform. There are also conflicting reports on the utility of some of the molecular techniques such as the use of rDNA for species identification within Sclerotinia. Freeman et al. (2002) reported that species within the Sclerotinaceae family possess almost identical rDNA sequences and, therefore, rDNA sequences are not useful for species differentiation within the genus Sclerotinia. Others have used SNP and group I introns to differentiate species within the genus Sclerotinia (Holst-Jensen et al. 1999; Njambere et al. 2008; Powers et al. 2001). During our 
initial studies to identify species of Sclerotinia infecting Kura clover, it became apparent that some of the published molecular techniques used were not reliable in distinguishing isolates collected in Poland.

The objective of our research was to develop molecular tools that would quickly and reliably distinguish between $S$. sclerotiorum and $S$. trifoliorum from different continents and host plants. These tools would be particularly useful in identifying Sclerotinia spp. that cause clover rot on Kura and other clover species.

\section{Materials and Methods}

Isolates of $\boldsymbol{S}$. trifoliorum and $\boldsymbol{S}$. sclerotiorum. In total, 40 isolates of $S$. trifoliorum (29 from Poland, 11 from the United States) and 55 isolates of S. sclerotiorum (26 from Poland, 29 from the United States) were obtained from surface-disinfested sclerotia or from host plant parts with symptoms indicative of clover rot (Table 1). Symptomatic plant parts were washed in tap water and surface disinfested for $1 \mathrm{~min}$ in $95 \%$ ethanol and $1 \mathrm{~min}$ in $0.825 \%$ sodium hypochlorite (The Clorox Company, Oakland, CA) prior to being plated onto potato dextrose agar (Fisher Scientific, Pittsburg) and incubated at 15 to $18^{\circ} \mathrm{C}$. Isolates were incubated on carrot discs to promote sclerotia formation and were maintained as dry sclerotia in plastic 15-ml tubes at $4^{\circ} \mathrm{C}$ for long-term storage. An isolate of $S$. trifoliorum (CBS: 122377) was obtained from the Centraalbureau voor Schimmelcultures (CBS) Fungal Biodiversity Centre in The Netherlands and used as a reference isolate for the species in this study.

DNA extraction. Isolates of Sclerotinia were grown in potato dextrose broth (EMD Chemicals, Inc., Gibbstown, NJ) for 7 to 10 days and the resulting mycelial culture was vacuum filtered and rinsed in sterile deionized water. DNA was extracted from mycelia using the FASTDNA SPIN Kit and the FastPrep Instrument (Qbiogene, Carlsbad, CA) according to the manufacturer's instructions. DNA quantity was estimated on a NanoDrop UV-Vis Spectrophotometer (Thermo Fisher Scientific, Inc., Waltham, MA). DNA was then diluted to $25 \mathrm{ng} \mu \mathrm{l}^{-1}$ for use as template for PCR. All DNA was stored at $-20^{\circ} \mathrm{C}$.

PCR primers and cycling conditions. Primers were used to amplify specific genes or gene regions (Table 2). ITS of the rDNA were amplified using primer pairs ITS1/ITS4 or ITS5/ITS4 (White et al. 1990). nSSU rDNA was amplified using primer pairs NS1/NS8 or NS5/NS6 (White et al. 1990). A portion of the $\beta$-tubulin gene was amplified using primer pairs TU1/TU2 (S. sclerotiorum isolates) and TU1/TU2/TU3 (S. trifoliorum isolates) (Vleugels et al. 2012). The aspartyl protease gene of $S$. sclerotiorum was amplified using the primer pair SSasprF/SSasprR (Abd-Elmagid et al. 2013). The calmodulin gene of $S$. trifoliorum was amplified using the published primer pair STCadF/STCadR (Abd-Elmagid et al. 2013). Primers (SScadF1/SScadR1) were designed for this study (PrimerQuest Software Package; Integrated DNA Technologies, Coralville, IA) to amplify the S. sclerotiorum calmodulin gene (Table 2). PCR were performed using GoTaq Green Master Mix (Promega Corp., Madison, WI) containing GoTaq DNA Polymerase in $1 \times$ Green GoTaq Reaction Buffer (pH 8.5), $200 \mu \mathrm{M}$ dNTP, $1.5 \mathrm{mM} \mathrm{MgCl}_{2}, 1 \mu \mathrm{M}$ each primer, and $2.5 \mu \mathrm{l}$ of DNA in a total volume of $25 \mu \mathrm{l} /$ reaction. PCR was performed using conditions specific for each target gene region in an Eppendorf Mastercycler Pro S programmable thermal cycler (Eppendorf AG, Hamburg, Germany). Conditions for amplification of ITS and 18S rDNA were denaturation at $94^{\circ} \mathrm{C}$ for $5 \mathrm{~min}$; followed by 35 cycles of $1 \mathrm{~min}$ of denaturation at $94^{\circ} \mathrm{C}$, $1 \mathrm{~min}$ of annealing at $52^{\circ} \mathrm{C}$, and $1 \mathrm{~min}$ of elongation at $72^{\circ} \mathrm{C}$; followed by a final elongation step at $72^{\circ} \mathrm{C}$ for $10 \mathrm{~min}$. Conditions for amplification of the $\beta$-tubulin gene were denaturation at $94^{\circ} \mathrm{C}$ for $5 \mathrm{~min}$; followed by 35 cycles of $30 \mathrm{~s}$ of denaturation at $94^{\circ} \mathrm{C}$, $30 \mathrm{~s}$ of annealing at $55^{\circ} \mathrm{C}$, and $1 \mathrm{~min}$ of elongation at $72^{\circ} \mathrm{C}$; followed by a final elongation step at $72^{\circ} \mathrm{C}$ for $5 \mathrm{~min}$. Conditions for amplification of the calmodulin and aspartyl protease genes were denaturation at $95^{\circ} \mathrm{C}$ for $2 \mathrm{~min}$; followed by 35 cycles of $30 \mathrm{~s}$ of denaturation at $95^{\circ} \mathrm{C}, 20 \mathrm{~s}$ of annealing at $61^{\circ} \mathrm{C}$, and $40 \mathrm{~s}$ of elongation at $72^{\circ} \mathrm{C}$; followed by a final elongation step at $72^{\circ} \mathrm{C}$ for $3 \mathrm{~min}$.
Proper negative controls (water blanks in place of template DNA) were used in all PCR experiments. PCR fragments were visualized by electrophoresis in $1 \times$ Tris-acetate-EDTA (University of Wisconsin-Madison) or $1 \times$ Tris-borate-EDTA (Poland) on 1.2 to $1.4 \%$ agarose gels containing SYBR Safe DNA gel stain (Life Technologies, Carlsbad, CA) using DNA size markers of either 1-kb ladder (Promega Corp.) or 100-kb ladder (EURx, Gdańsk, Poland) to estimate the size of the fragments.

Sequence analysis and dendrogram construction. In order to obtain sequence data for isolate comparisons and to determine the presence or absence of introns and SNP within the rDNA of the isolates examined, PCR fragments were purified using the Wizard SV Gel and PCR Clean-Up System (Promega Corp.). Sequencing was performed using ABI 3730xl DNA Analyzers (Applied Biosystems, Foster City, CA) at the University of Wisconsin-Madison Biotechnology Center or at Genomed (Warszawa, Poland). Sequence comparisons were carried out using BLASTn on GenBank. Alignments were carried out using ClustalW through the Molecular Evolutionary Genetics Analysis software package, version 6 (MEGA6) (Tamura et al. 2013). A dendrogram was constructed from ITS rDNA sequences using the maximum-likelihood algorithm (MEGA6 software) (Tamura et al. 2013). The Kimura two-parameter model was applied and bootstrap analysis with 1,000 replications was done to assess group support. rDNA sequences obtained from isolates examined in this study were analyzed and compared with reference sequences obtained from the National Center for Biotechnology Information (NCBI) to construct the dendrogram.

\section{Results}

SNP in the ITS region. Amplification with primers ITS1 and ITS4 resulted in a 540-bp product with all isolates tested (24 S. sclerotiorum and $26 \mathrm{~S}$. trifoliorum), regardless of species. Sequencing of both strands revealed SNP at two separate positions (120 and 376). All S. sclerotiorum isolates sequenced, regardless of origin, had thymine at both positions, which was previously reported to be typical for the species (Njambere et al. 2008) (Table 1). Five of the Polish $S$. trifoliorum isolates sequenced and the $S$. trifoliorum reference isolate CBS: 122377 had guanine (G) at position 120 and cytosine at position 376, which was previously reported to be typical for $S$. trifoliorum (Njambere et al. 2008) (Table 1). In all, 8 of 13 Polish isolates and all $11 \mathrm{~S}$. trifoliorum isolates from the United States that were sequenced had thiamine (T) at position 120 and cytosine (C) at position 376 (Table 1). Sequencing of both strands was performed twice to ensure that no errors were made.

Presence of introns in rRNA. Amplification of the isolates with primers ITS5 and ITS4 resulted in an approximately 560-bp product with all isolates of $S$. sclerotiorum (regardless of origin) and with 10 of the Polish and all U.S. isolates of S. trifoliorum. Nineteen S. trifoliorum isolates from Poland and the reference isolate (CBS:122377) all yielded a product approximately $1,000 \mathrm{bp}$ in size, indicating the presence of at least one intron occurring in the region between the ITS1 and ITS5 primer annealing sites in these $S$. trifoliorum isolates (Powers et al. 2001).

Amplification of the nSSU rDNA with primers NS5 and NS6 revealed the presence of introns in all $S$. trifoliorum isolates tested, with the exception of Polish isolate St1513TR and the S. trifoliorum reference isolate CBS:122377 (data not shown).

Amplification of the nSSU rDNA with primers NS1 and NS8 revealed the presence of multiple introns or introns of varying sizes in all S. trifoliorum isolates examined, regardless of origin. All of the products obtained from the U.S. isolates tested were of the same size; however, there were at least three different size polymorphisms among the Polish isolates examined (Table 1; Fig. 1). Three polymorphisms of distinct lengths were revealed among the S. trifoliorum isolates. In total, 10 of the Polish S. trifoliorum and all of the U.S. S. trifoliorum isolates examined yielded a product approximately 2,000 bp in size (group I), only 2 isolates of $S$. trifoliorum (St1513TR and CBS 122377) yielded a product approximately $2,200 \mathrm{bp}$ in size (group II), and 18 Polish S. trifoliorum isolates 
yielded a product approximately $2,500 \mathrm{bp}$ in size (group III). No introns were detected in any of the S. sclerotiorum isolates examined.

Amplification of the $\boldsymbol{\beta}$-tubulin gene. PCR with primers TU1/TU2/ TU3 amplified a single DNA fragment (494 bp) with all S. sclerotiorum isolates examined and two DNA fragments (494 and $390 \mathrm{bp}$ ) with all S. trifoliorum isolates examined (Table 1; Fig. 2).

Amplification of calmodulin and aspartyl protease genes. PCR with primers STCadF/STCadR and SSasprF/SSasprR resulted in amplicons for all isolates examined, regardless of species. For isolates originating

Table 1. Origin of Sclerotinia trifoliorum and S. sclerotiorum isolates and results of polymerase chain reaction (PCR) assays and analyses on ribosomal DNA for species identification $^{\mathrm{a}}$

\begin{tabular}{|c|c|c|c|c|c|c|c|c|c|c|c|c|c|c|}
\hline \multirow[b]{2}{*}{ Code $^{f}$} & \multirow[b]{2}{*}{ Host $^{\mathrm{g}}$} & \multirow[b]{2}{*}{ Year $^{\text {h }}$} & \multirow[b]{2}{*}{ Loc $^{\mathrm{i}}$} & \multicolumn{2}{|c|}{ SNPb } & \multirow[b]{2}{*}{ ITS $^{\mathbf{j}}$} & \multicolumn{2}{|c|}{ SSU $^{\mathbf{c}}$} & \multirow[b]{2}{*}{ Bands ${ }^{k}$} & \multicolumn{3}{|c|}{ Product $^{d}$} & \multicolumn{2}{|c|}{ Acc. number ${ }^{\mathrm{e}}$} \\
\hline & & & & 120 & 376 & & NS5/6 & NS1/8 & & STC & SSas & Ssca & NCBI & CBS \\
\hline \multicolumn{15}{|l|}{ Polish } \\
\hline \multicolumn{15}{|l|}{ S. trifoliorum } \\
\hline St 0111 TA & Trifolium ambiguum & 2011 & KP & $\mathrm{T}$ & $\mathrm{C}$ & - & + & I & + & + & + & - & $\ldots$ & $\ldots$ \\
\hline St 0211 TA & T. ambiguum & 2011 & $\mathrm{KP}$ & $\mathrm{T}$ & $\mathrm{C}$ & - & + & I & + & + & + & - & JQ743329 & $\ldots$ \\
\hline St 0411 TA & T. ambiguum & 2011 & $\mathrm{KP}$ & $\mathrm{T}$ & $\mathrm{C}$ & - & + & I & + & + & + & - & $\ldots$ & $\ldots$ \\
\hline St 0511 TA & T. ambiguum & 2011 & $\mathrm{KP}$ & $\mathrm{T}$ & $\mathrm{C}$ & - & + & I & + & + & + & - & КT970795 & 133234 \\
\hline St 0612 TA & T. ambiguum & 2012 & $\mathrm{KP}$ & G & $\mathrm{C}$ & + & + & III & + & + & + & - & $\ldots$ & $\ldots$ \\
\hline St 0712 TA & T. ambiguum & 2012 & $\mathrm{KP}$ & $\mathrm{T}$ & $\mathrm{C}$ & - & + & I & + & + & + & - & $\ldots$ & $\ldots$ \\
\hline St 0812 TA & T. ambiguum & 2012 & $\mathrm{KP}$ & $\mathrm{T}$ & $\mathrm{C}$ & - & + & I & + & + & + & - & KT224641 & $\ldots$ \\
\hline St 0912 TA & T. ambiguum & 2012 & $\mathrm{KP}$ & $\mathrm{G}$ & $\mathrm{C}$ & + & + & III & + & + & + & - & $\ldots$ & $\ldots$ \\
\hline St 1412 TA & T. ambiguum & 2012 & $\mathrm{KP}$ & G & $\mathrm{C}$ & + & + & III & + & + & + & - & KT224642 & $\ldots$ \\
\hline St 1513 TR & T. repens & 2013 & $\mathrm{KP}$ & $\mathrm{T}$ & $\mathrm{C}$ & + & - & II & + & + & + & - & KT224643 & 140899 \\
\hline St 1613 TR & T. repens & 2013 & $\mathrm{KP}$ & l & I & + & + & III & + & + & + & - & $\ldots$ & $\ldots$ \\
\hline St 1713 TR & T. repens & 2013 & $\mathrm{KP}$ & l & l & + & + & III & + & + & + & - & $\ldots$ & $\ldots$ \\
\hline St 1813 TP & T. pratense & 2013 & $\mathrm{KP}$ & $\mathrm{T}$ & $\mathrm{C}$ & - & + & I & + & + & + & - & КT986229 & 140900 \\
\hline St 1913 TP & T. pratense & 2013 & $\mathrm{KP}$ & l & l & - & + & I & + & + & + & - & $\ldots$ & $\ldots$ \\
\hline St 2013 TP & T. pratense & 2013 & $\mathrm{KP}$ & l & l & + & + & III & + & + & + & - & $\ldots$ & $\ldots$ \\
\hline St 2113 TRe & T. resupinatum & 2013 & $\mathrm{KP}$ & G & $\mathrm{C}$ & + & + & III & + & + & + & - & KT224644 & 140901 \\
\hline St 2213 TR & T. repens & 2013 & $\mathrm{KP}$ & l & / & + & + & III & + & + & + & - & $\ldots$ & $\ldots$ \\
\hline St 2313 TR & T. repens & 2013 & $\mathrm{KP}$ & I & I & + & + & III & + & + & + & - & $\ldots$ & $\ldots$ \\
\hline St 2413 TA & T. ambiguum & 2013 & $\mathrm{P}$ & G & $\mathrm{C}$ & + & + & III & + & + & + & - & КT986230 & 140902 \\
\hline St 2513 TA & T. ambiguum & 2013 & $\mathrm{P}$ & l & I & + & + & III & + & + & + & - & $\ldots$ & $\ldots$ \\
\hline St 2613 TA & T. ambiguum & 2013 & $\mathrm{P}$ & I & I & - & + & I & + & + & + & - & $\ldots$ & $\ldots$ \\
\hline St 2713 TA & T. ambiguum & 2013 & $\mathrm{P}$ & I & I & + & + & III & + & + & + & - & $\ldots$ & $\ldots$ \\
\hline St 2813 TR & T. repens & 2013 & $\mathrm{P}$ & I & I & + & + & III & + & + & + & - & $\ldots$ & $\ldots$ \\
\hline St 2913 TR & T. repens & 2013 & $\mathrm{P}$ & l & l & + & + & III & + & + & + & - & $\ldots$ & $\ldots$ \\
\hline St 3013 TR & T. repens & 2013 & $\mathrm{P}$ & l & l & + & + & III & + & + & + & - & $\ldots$ & $\ldots$ \\
\hline St 3113 TP & T. pratense & 2013 & $\mathrm{P}$ & l & I & + & + & III & + & + & + & - & $\ldots$ & $\ldots$ \\
\hline St 3213 TP & T. pratense & 2013 & $\mathrm{P}$ & l & l & + & + & III & + & + & + & - & $\ldots$ & $\ldots$ \\
\hline St 3313 TR & T. repens & 2013 & $\mathrm{P}$ & l & l & - & + & I & + & + & + & - & $\ldots$ & $\ldots$ \\
\hline St 3413 TR & T. repens & 2013 & $\mathrm{P}$ & I & I & + & + & III & + & + & + & - & $\ldots$ & $\ldots$ \\
\hline \multicolumn{15}{|c|}{ S. sclerotiorum } \\
\hline Ss $0311 \mathrm{BN}$ & Brassica napus & 2011 & $\mathrm{KP}$ & $\mathrm{T}$ & $\mathrm{T}$ & - & - & 0 & - & + & + & + & КT970791 & $\ldots$ \\
\hline Ss 1012 HA & Helichrysum arenarium & 2012 & $\mathrm{KP}$ & $\mathrm{T}$ & $\mathrm{T}$ & - & - & 0 & - & + & + & + & $\ldots$ & $\ldots$ \\
\hline Ss 1112 HA & H. arenarium & 2012 & $\mathrm{KP}$ & $\mathrm{T}$ & $\mathrm{T}$ & - & - & 0 & - & + & + & + & $\ldots$ & $\ldots$ \\
\hline Ss $1212 \mathrm{HA}$ & H. arenarium & 2012 & $\mathrm{KP}$ & $\mathrm{T}$ & $\mathrm{T}$ & - & - & 0 & - & + & + & + & KT224645 & 140893 \\
\hline Ss 1312 DC & D. carota & 2012 & $\mathrm{KP}$ & $\mathrm{T}$ & $\mathrm{T}$ & - & - & 0 & - & + & + & + & KT224646 & $\ldots$ \\
\hline Ss 3513 BN & B. napus & 2013 & $\mathrm{KP}$ & $\mathrm{T}$ & $\mathrm{T}$ & - & - & 0 & - & + & + & + & KT224647 & 140894 \\
\hline Ss 3613 BN & B. napus & 2013 & $\mathrm{KP}$ & l & l & - & - & 0 & - & + & + & + & $\ldots$ & $\ldots$ \\
\hline Ss $3713 \mathrm{BN}$ & B. napus & 2013 & $\mathrm{KP}$ & l & l & - & - & 0 & - & + & + & + & $\ldots$ & $\ldots$ \\
\hline Ss 3813 BN & B. napus & 2013 & $\mathrm{KP}$ & l & l & - & - & 0 & - & + & + & + & $\ldots$ & $\ldots$ \\
\hline Ss 3913 BN & B. napus & 2013 & $\mathrm{KP}$ & l & l & - & - & 0 & - & + & + & + & $\ldots$ & $\ldots$ \\
\hline Ss 4013 BN & B. napus & 2013 & $\mathrm{KP}$ & I & l & - & - & 0 & - & + & + & + & $\ldots$ & $\ldots$ \\
\hline Ss $4113 \mathrm{BN}$ & B. napus & 2013 & $\mathrm{KP}$ & I & I & - & - & 0 & - & + & + & + & $\ldots$ & $\ldots$ \\
\hline \multirow[t]{2}{*}{ Ss $4213 \mathrm{BN}$} & B. napus & 2013 & $\mathrm{KP}$ & I & 1 & - & - & 0 & - & + & + & + & $\ldots$ & $\ldots$ \\
\hline & & & & & & & & & & & & \multicolumn{3}{|c|}{ (continued on next page) } \\
\hline
\end{tabular}

a Symbols: / indicates not analyzed and + or - indicate the presence or absence, respectively, of the PCR product.

${ }^{\mathrm{b}}$ Presence of single-nucleotide polymorphism (SNP) reaction with internal transcribed spacer (ITS)1/ITS4 at positions 120 or 376.

$\mathrm{c}$ Presence of introns in small subunit (SSU) reaction with NS5/6 and NS1/8. Length of product with NS1/NS8: 0 indicates no introns and I to III indicate groups of isolates with introns of different size.

d Product with STCadF/STCadR (STC), SSasprF/SSasprR (SSas), and SscadF1/SscadR1 (Ssca).

e Accession numbers in the Centraalbureau voor Schimmelcultures (CBS) and National Center for Biotechnology Information (NCBI).

${ }^{\mathrm{f}}$ Codes for Polish and U.S. isolates of S. trifoliorum and S. sclerotiorum and control samples (Control).

g Host plant.

h Year of collection.

${ }^{\mathrm{i}}$ Location of collection in Polish provinces (KP $=$ Kuyavia-Pomerania, $\mathrm{P}=$ Pomerania, and LS $=$ Lower Silesia), and states of the United States (IL $=$ Illinois, $\mathrm{MI}=$ Michigan, $\mathrm{MN}=$ Minnesota, and WI = Wisconsin), and The Netherlands (NL).

j Presence of introns in reaction with ITS5/4.

k Presence of two bands in reaction with TU1/TU2/TU3.

${ }^{1}$ S. trifoliorum from the soil under Trifolium sp.

m ITS sequences of S. trifoliorum and S. sclerotiorum. 
from Poland, the intensity of the band visualized on the gel with primers STCadF/STCadR was greater for all S. trifoliorum isolates in comparison with the $S$. sclerotiorum isolates (Fig. 3). In contrast, the intensity of the band visualized on the gel with primers SSasprF/ SSasprR was greater for all S. sclerotiorum isolates in comparison with the $S$. trifoliorum isolates. The primer pair SScadF1/SScadR1 amplified a single fragment ( $100 \mathrm{bp})$ in all of the S. sclerotiorum isolates tested but in none of the $S$. trifoliorum isolates tested (Fig. 4).

Dendrogram based on rDNA sequences. DNA sequences obtained during the course of this research from fragments of the

Table 1. (continued from preceding page)

\begin{tabular}{|c|c|c|c|c|c|c|c|c|c|c|c|c|c|c|}
\hline \multirow[b]{2}{*}{ Code $^{f}$} & \multirow[b]{2}{*}{ Host $^{\mathrm{g}}$} & \multirow[b]{2}{*}{ Yearh } & \multirow[b]{2}{*}{$\operatorname{Loc}^{\mathrm{i}}$} & \multicolumn{2}{|c|}{$\mathbf{S N P}^{\mathbf{b}}$} & \multirow[b]{2}{*}{ ITS $^{\mathbf{j}}$} & \multicolumn{2}{|c|}{$\mathbf{S S U}^{\mathbf{c}}$} & \multirow[b]{2}{*}{ Bands $^{k}$} & \multicolumn{3}{|c|}{ Product $^{\mathrm{d}}$} & \multicolumn{2}{|c|}{ Acc. number ${ }^{\mathrm{e}}$} \\
\hline & & & & 120 & 376 & & NS5/6 & NS1/8 & & STC & SSas & Ssca & NCBI & CBS \\
\hline Ss 4313 BN & B. napus & 2013 & $\mathrm{KP}$ & I & I & - & - & 0 & - & + & + & + & $\ldots$ & $\ldots$ \\
\hline Ss 4413 BN & B. napus & 2013 & $\mathrm{KP}$ & I & I & - & - & 0 & - & + & + & + & $\ldots$ & $\ldots$ \\
\hline Ss $4513 \mathrm{BN}$ & B. napus & 2013 & $\mathrm{KP}$ & I & I & - & - & 0 & - & + & + & + & $\ldots$ & $\ldots$ \\
\hline Ss 4613 BN & B. napus & 2013 & $\mathrm{KP}$ & I & I & - & - & 0 & - & + & + & + & $\ldots$ & $\ldots$ \\
\hline Ss 4713 DC & D. carota & 2013 & $\mathrm{KP}$ & $\mathrm{T}$ & $\mathrm{T}$ & - & - & 0 & - & + & + & + & $\ldots$ & $\ldots$ \\
\hline Ss 4812 AG & Apium graveolens & 2012 & LS & $\mathrm{T}$ & $\mathrm{T}$ & - & - & 0 & - & + & + & + & KT224648 & 140895 \\
\hline Ss 4912 DC & D. carota & 2012 & LS & I & I & - & - & 0 & - & + & + & + & $\ldots$ & $\ldots$ \\
\hline Ss 5012 DC & D. carota & 2012 & LS & I & I & - & - & 0 & - & + & + & + & $\ldots$ & $\ldots$ \\
\hline Ss 5112 PC & Petroselinum crispum & 2012 & $\mathrm{LS}$ & $\mathrm{T}$ & $\mathrm{T}$ & - & - & 0 & - & + & + & + & KT224649 & 140896 \\
\hline Ss 5213 LS & Lactuca sativa & 2013 & LS & $\mathrm{T}$ & $\mathrm{T}$ & - & - & 0 & - & + & + & + & KT224650 & 140897 \\
\hline Ss 5314 DC & D. carota & 2014 & $\mathrm{KP}$ & $\mathrm{T}$ & $\mathrm{T}$ & - & - & 0 & - & + & + & + & KT970792 & 141019 \\
\hline Ss 5415 BO & B. oleracea var. italica & 2015 & $\mathrm{KP}$ & $\mathrm{T}$ & $\mathrm{T}$ & - & - & 0 & - & + & + & + & КT970793 & 140898 \\
\hline Ss 5515 BO & B. oleracea var. italica & 2015 & $\mathrm{KP}$ & I & / & - & - & 0 & - & + & + & + & $\ldots$ & $\ldots$ \\
\hline \multicolumn{15}{|l|}{ U.S. } \\
\hline S. trifoliorum & & & & & & & & & & & & & & \\
\hline St 01 TP & T. pratense & 1996 & WI & $\mathrm{T}$ & $\mathrm{C}$ & - & + & I & + & + & + & - & $\ldots$ & $\ldots$ \\
\hline St $02 \mathrm{TP}$ & T. pratense & 1997 & WI & $\mathrm{T}$ & $\mathrm{C}$ & - & + & I & + & + & + & - & KT224651 & $\ldots$ \\
\hline St 03 TP & T. pratense & 1992 & WI & $\mathrm{T}$ & $\mathrm{C}$ & - & + & I & + & + & + & - & KT224652 & $\ldots$ \\
\hline St 04 TP & T. pratense & 1996 & WI & $\mathrm{T}$ & $\mathrm{C}$ & - & + & I & + & + & + & - & $\ldots$ & $\ldots$ \\
\hline St 05 TP & T. pratense & 1995 & WI & $\mathrm{T}$ & $\mathrm{C}$ & - & + & I & + & + & + & - & $\ldots$ & $\ldots$ \\
\hline St 06 TP & T. pratense & 1992 & WI & $\mathrm{T}$ & $\mathrm{C}$ & - & + & I & + & + & + & - & $\ldots$ & $\ldots$ \\
\hline St 07 TP & T. pratense & 1992 & WI & $\mathrm{T}$ & $\mathrm{C}$ & - & + & I & + & + & + & - & $\ldots$ & $\ldots$ \\
\hline St $08 \mathrm{TP}$ & T. pratense & 1994 & WI & $\mathrm{T}$ & $\mathrm{C}$ & - & + & I & + & + & + & - & $\ldots$ & $\ldots$ \\
\hline St 09 TP & T. pratense & 1997 & WI & $\mathrm{T}$ & $\mathrm{C}$ & - & + & I & + & + & + & - & $\ldots$ & $\ldots$ \\
\hline St $10 \mathrm{TP}$ & T. pratense & 1993 & WI & $\mathrm{T}$ & $\mathrm{C}$ & - & + & I & + & + & + & - & $\ldots$ & $\ldots$ \\
\hline St $11 \mathrm{TP}$ & T. pratense & 1994 & WI & $\mathrm{T}$ & $\mathrm{C}$ & - & + & I & + & + & + & - & $\ldots$ & $\ldots$ \\
\hline S. sclerotiorum & & & & & & & & & & & & & & \\
\hline Ss 12 GM & Glycine $\max$ & 2012 & WI & $\mathrm{T}$ & $\mathrm{T}$ & - & - & 0 & - & + & + & + & KT224653 & $\ldots$ \\
\hline Ss 13 GM & G. $\max$ & 2012 & WI & I & I & - & - & 0 & - & + & + & + & $\ldots$ & $\ldots$ \\
\hline Ss $14 \mathrm{BN}$ & B. napus & Unknown & $\mathrm{IL}$ & $\mathrm{T}$ & $\mathrm{T}$ & - & - & 0 & - & + & + & + & KT224654 & $\ldots$ \\
\hline Ss 15 GM & G. $\max$ & Unknown & $\mathrm{IL}$ & $\mathrm{T}$ & $\mathrm{T}$ & - & - & 0 & - & + & + & + & KT224655 & $\ldots$ \\
\hline Ss 16 AT & Abutilon theophrasti & Unknown & IL & $\mathrm{T}$ & $\mathrm{T}$ & - & - & 0 & - & + & + & + & KT224656 & $\ldots$ \\
\hline Ss 17 GM & G. $\max$ & Unknown & MI & $\mathrm{T}$ & $\mathrm{T}$ & - & - & 0 & - & + & + & + & $\ldots$ & $\ldots$ \\
\hline Ss 18 GM & G. $\max$ & Unknown & MI & I & / & - & - & 0 & - & + & + & + & $\ldots$ & $\ldots$ \\
\hline Ss 19 GM & G. $\max$ & 2000 & WI & $\mathrm{T}$ & $\mathrm{T}$ & - & - & 0 & - & + & + & + & $\ldots$ & $\ldots$ \\
\hline Ss $20 \mathrm{GM}$ & G. $\max$ & Unknown & WI & I & I & - & - & 0 & - & + & + & + & $\ldots$ & $\ldots$ \\
\hline Ss $21 \mathrm{GM}$ & G. $\max$ & Unknown & WI & I & I & - & - & 0 & - & + & + & + & $\ldots$ & $\ldots$ \\
\hline Ss 22 GM & G. $\max$ & 2012 & WI & I & I & - & - & 0 & - & + & + & + & $\ldots$ & $\ldots$ \\
\hline Ss 23 GM & G. $\max$ & 2012 & WI & I & I & - & - & 0 & - & + & + & + & $\ldots$ & $\ldots$ \\
\hline Ss 24 GM & G. $\max$ & 2012 & WI & I & I & - & - & 0 & - & + & + & + & $\ldots$ & $\ldots$ \\
\hline Ss 25 GM & G. $\max$ & Unknown & WI & I & I & - & - & 0 & - & + & + & + & $\ldots$ & $\ldots$ \\
\hline Ss 26 GM & G. $\max$ & 2003 & WI & $\mathrm{T}$ & $\mathrm{T}$ & - & - & 0 & - & + & + & + & $\ldots$ & $\ldots$ \\
\hline Ss 27 GM & G. $\max$ & 2012 & WI & I & I & - & - & 0 & - & + & + & + & $\ldots$ & $\ldots$ \\
\hline Ss $28 \mathrm{GM}$ & G. $\max$ & 2012 & WI & I & I & - & - & 0 & - & + & + & + & $\ldots$ & $\ldots$ \\
\hline Ss 29 GM & G. $\max$ & 2000 & WI & $\mathrm{T}$ & $\mathrm{T}$ & - & - & 0 & - & + & + & + & KT224657 & $\ldots$ \\
\hline Ss 30 GM & G. $\max$ & Unknown & WI & I & I & - & - & 0 & - & + & + & + & $\ldots$ & $\ldots$ \\
\hline Ss $31 \mathrm{GM}$ & G. $\max$ & 2012 & WI & I & I & - & - & 0 & - & + & + & + & $\ldots$ & $\ldots$ \\
\hline Ss 32 GM & G. $\max$ & 2012 & WI & I & I & - & - & 0 & - & + & + & + & $\ldots$ & $\ldots$ \\
\hline Ss 33 GM & G. $\max$ & Unknown & WI & I & I & - & - & 0 & - & + & + & + & $\ldots$ & $\ldots$ \\
\hline Ss 34 GM & G. $\max$ & 2012 & WI & I & I & - & - & 0 & - & + & + & + & $\ldots$ & $\ldots$ \\
\hline Ss 35 GM & G. $\max$ & Unknown & WI & I & I & - & - & 0 & - & + & + & + & $\ldots$ & $\ldots$ \\
\hline Ss 36 GM & G. $\max$ & 2012 & WI & I & I & - & - & 0 & - & + & + & + & $\ldots$ & $\ldots$ \\
\hline Ss $37 \mathrm{PV}$ & Phaseolus vulgaris & 2009 & WI & $\mathrm{T}$ & $\mathrm{T}$ & - & - & 0 & - & + & + & + & KT224658 & $\ldots$ \\
\hline Ss $38 \mathrm{P}$ & Petunia sp. & 2009 & $\mathrm{MN}$ & $\mathrm{T}$ & $\mathrm{T}$ & - & - & 0 & - & + & + & + & KT224659 & $\ldots$ \\
\hline Ss 39 GM & G. $\max$ & 2013 & MN & $\mathrm{T}$ & $\mathrm{T}$ & - & - & 0 & - & + & + & + & KT224660 & $\ldots$ \\
\hline Ss 40 GM & G. $\max$ & 2013 & $\mathrm{MN}$ & I & I & - & - & 0 & - & + & + & + & $\ldots$ & $\ldots$ \\
\hline Control & & & & & & & & & & & & & & \\
\hline CBS: 122377 & Soil $^{1}$ & 2007 & NL & G & $\mathrm{C}$ & + & - & II & + & + & + & - & КT970794 & 122377 \\
\hline EU082464 & ITS S. trif ${ }^{\mathrm{m}}$ & I & I & $\mathrm{G}$ & $\mathrm{C}$ & + & + & I & I & I & I & I & EU082464 & $\ldots$ \\
\hline EU082466 & ITS S. scler ${ }^{\mathrm{m}}$ & I & I & $\mathrm{T}$ & $\mathrm{T}$ & - & - & I & I & I & I & I & EU082466 & $\ldots$ \\
\hline
\end{tabular}


ITS rDNA (amplicons from primers ITS1 and ITS4) from a subset of isolates (25 S. trifoliorum isolates, including CBS 122377, and $23 \mathrm{~S}$. sclerotiorum isolates) were used to construct a dendrogram using the maximum-likelihood algorithm. Sequences obtained from NCBI for six $S$. trifoliorum and six $S$. sclerotiorum isolates were used for reference and comparison purposes (Fig. 5, accession numbers). In addition, sequences from S. glacialis, S. homoeocarpa, S. minor, $S$. tetraspora, Dumontinia tuberosa, and Botrytis cinerea were included for additional comparison (Fig. 5, accession numbers). The resulting dendrogram demonstrated that $S$. sclerotiorum, S. minor, and S. trifoliorum are closely related and all grouped into a single clade; however, there was a clear grouping within the clade by species (Fig. 5). All $S$. sclerotiorum isolates fell into a single group. Isolates of $S$. trifoliorum grouped into a large subclade comprising three distinct groups of isolates. The first group comprised five isolates (all Polish) and four of the NCBI reference isolates, all of which possess $\mathrm{G} / \mathrm{C}$ at positions 120/376 in the ITS sequence. A second, larger group comprised 19 isolates (11 U.S. isolates and 9 Polish isolates) and 2 of the NCBI reference isolates, all of which possess T/C at positions 120/ 376 in the ITS sequence. Isolate CBS:122377, the sole member of the third group, possesses G/C at positions 120/376, which is typical for S. trifoliorum (Njambere et al. 2008) but differs from the other $S$. trifoliorum isolates by five other nucleotide substitutions (sequence data deposited in NCBI; Table 1). All isolates were sequenced twice to ensure the validity of the data. The overall mean distance of the rDNA ITS sequences was computed using the Mega 6 software package. The diversity of the 70 analyzed sequences (representing three genera and eight distinct species) was $1.6 \%$, whereas the diversity of the rDNA ITS sequences of only the Polish and U.S. isolates of S. trifoliorum and S. sclerotiorum was $0.2 \%$, indicating a high degree of similarity.

\section{Discussion}

The use of SNP and the presence of introns within the rDNA were useful to differentiate between S. sclerotiorum and S. trifoliorum. Using SNP or intron analyses techniques, no variation in the rDNA of $S$. sclerotiorum was identified in the collection of isolates in this study. However, using SNP combined with the presence or absence of introns, we detected intraspecies variation within S. trifoliorum. SNP analyses revealed two groups of $S$. trifoliorum isolates among the isolates included in our study. One group of five Polish isolates and the S. trifoliorum reference isolate (CBS:122377) all possessed the SNP G/C at positions $120 / 376$ within the ITS region of the rDNA sequence, which has been reported to be typical for S. trifoliorum (Njambere et al. 2008). Interestingly, these same isolates were placed into either group II or III based on product size and presence of introns within nSSU rDNA (amplification with primers NS1/NS8). A second group of $S$. trifoliorum isolates in our study, comprising 8 Polish isolates and all 11 U.S. isolates, possessed the SNP T/C at positions 120/376. All of these isolates, with one exception (St1513TR), were placed into group I based on product size and presence of introns within nSSU rDNA (amplification with primers NS1/ NS8). Njambere et al. (2008) previously reported that all of the S. trifoliorum isolates from chickpea in California used in their study possessed G/C at positions 120/376. Yli-Mattila et al. (2010) examined a small number of $S$. trifoliorum isolates from Finland and detected the SNP T/C at positions 120/376 in the ITS rDNA region in at least two of their isolates from red clover. All 11 of the isolates used in our study from red clover from Wisconsin possessed T/C at the same positions within the ITS rDNA region analyzed.

Using the primers NS1 and NS8 to amplify the nSSU rDNA, we were able to differentiate between $S$. sclerotiorum and $S$. trifoliorum isolates based on amplicon sizes. All isolates of $S$. sclerotiorum used in this study produced a single amplicon with no length variations, whereas multiple introns or introns of varying sizes were found in all S. trifoliorum isolates examined, resulting in amplicons of varying

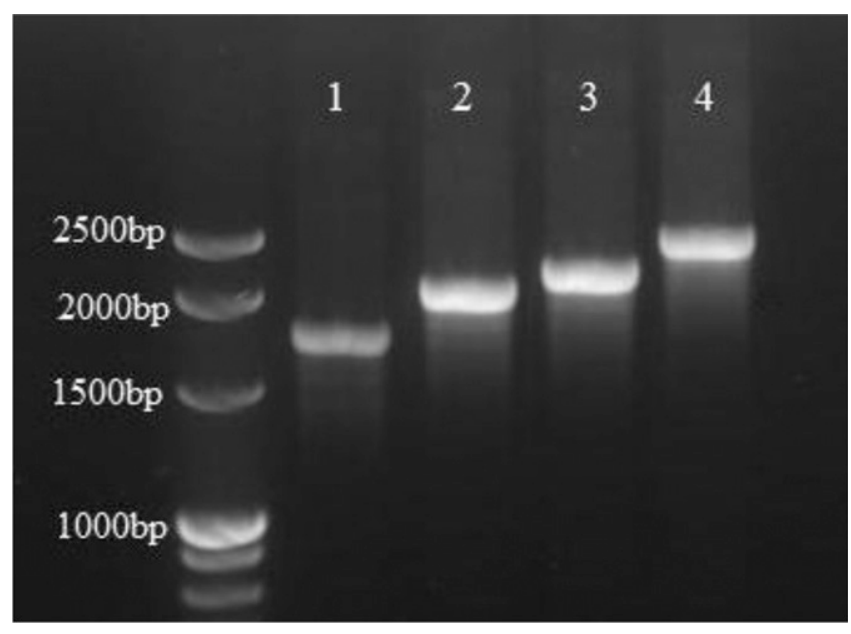

Fig. 1. Products of polymerase chain reaction amplification of Sclerotinia trifoliorum and S. sclerotiorum isolates using the primers NS1 and NS8. A product of approximately $1,750 \mathrm{bp}$ in size (no introns) was identified in $S$. sclerotiorum isolate Ss5314 DC (lane 1) and larger products of different sizes in S. trifoliorum St 1813 TP with introns group I (lane 2), St 1513 TR with introns group II (lane 3), and St 0912 TA with introns group III (lane 4).

Table 2. Targets, primer code, primer sequence, and expected size of polymerase chain reaction products amplified in this study

\begin{tabular}{|c|c|c|c|}
\hline Target $^{\mathbf{a}}$ & Primer code & Sequence $5^{\prime} \rightarrow 3^{\prime}$ & Expected size (bp) \\
\hline ITS region ${ }^{b}$ & ITS1/ITS4 & TCCGTAGGTGAACCTGCGG TCCTCCGCTTATTGATATGC & 540 \\
\hline ITS region ${ }^{\mathrm{b}}$ & ITS5/ITS4 & TCCTCCGCTTATTGATATGC GGAAGTAAAAGTCGTAACAAGG & $\begin{array}{l}560(\text { S. sclerotiorum }) \\
1,000 \text { (S. trifoliorum })\end{array}$ \\
\hline $18 \mathrm{~S}^{\mathrm{b}}$ & NS5/NS6 & AACTTAAAGGAATTGACGGAAG GCATCACAGACCTGTTATTGCCTC & $\begin{array}{l}250(\text { S. sclerotiorum }) \\
600(\text { S. trifoliorum })\end{array}$ \\
\hline $18 \mathrm{~S}^{\mathrm{c}}$ & NS1/NS8 & GTAGTCATATGCTTGTCTC TCCGCAGGTTCACCTACGGA & $\begin{array}{l}1,750(\text { S. sclerotiorum }) \\
>1,750(\text { S. trifoliorum })\end{array}$ \\
\hline \multicolumn{4}{|l|}{ Gene } \\
\hline$\beta$-Tubulin ${ }^{\mathrm{d}}$ & TU1/TU2/TU3 & $\begin{array}{l}\text { CCTGAAAAGCACCCCACTAT ACGGCACGAGGAACATACTT } \\
\text { AACTCAACTCGACCGATGCT }\end{array}$ & $\begin{array}{l}494 \text { (S. sclerotiorum and } \\
\text { S. trifoliorum), } \\
390(\text { S. trifoliorum })\end{array}$ \\
\hline Calmoduline & STCadF/STCadR & TCCTAGATCGACTCTCCTCCTTT CGTGTTATTGCCTCCTTGTTG & 97 \\
\hline Aspartyl protease $\mathrm{e}^{\mathrm{e}}$ & SSasprF/SSasprR & CATTGGAAGTCTCGTCGTCA TCAAACGCCAAAGCTGTATG & 171 \\
\hline Calmodulin ${ }^{\mathrm{f}}$ & SscadF1/SscadR1 & TGTCCCAGTTCGACTCTCCTCT TGTTATTGCCCCCTTTGTTGGT & 100 \\
\hline
\end{tabular}

a ITS = internal transcribed spacer.

${ }^{\mathrm{b}}$ Njambere et al. (2008) and White et al. (1990).

${ }^{c}$ Powers et al. (2001) and White et al. (1990).

d Vleugels et al. (2012).

e Abd-Elmagid et al. (2013).

${ }^{\mathrm{f}}$ New primers designed in this research. 
sizes. We detected that there are polymorphisms of at least three distinct lengths among isolates within $S$. trifoliorum, with the most variability within the Polish isolates. All U.S. S. trifoliorum isolates produced the same-sized amplicon when gene targets were amplified with the primer pairs NS1/NS8 and NS5/NS6. However, at least three polymorphisms of distinct lengths were revealed with amplification of the Polish isolates with primers NS1/NS8. The high level of intraspecific variation among isolates of $S$. trifoliorum detected by the presence or absence of nSSU rDNA introns was previously reported (Njambere et al. 2008; Powers et al. 2001). Our results support the previous findings. Interestingly, most of the variation among isolates was detected within the Polish isolates we tested. These isolates came from at least four host species and two locations collected over a period of 3 years. There was no apparent variation among the U.S. isolates tested in this study, which could be attributed to the fact that all of the U.S. isolates were collected from the same host species (red clover) and same location (Wisconsin) over a span of several years. A more thorough testing of U.S. populations of S. trifoliorum utilizing isolates from a broader host range and from various geographical locations within the United States would be necessary to make a more accurate description of the intraspecific variability among isolates of S. trifoliorum present there.

S. trifoliorum is a heterothallic fungus (F and S mating types) and, therefore, is capable of sexual reproduction and outcrossing as well as vegetative reproduction (Vleugels et al. 2012). The genetic variation we observed within the Polish isolates in our study may be an indication of genetic mixing occurring in populations of
S. trifoliorum in Poland. S. sclerotiorum, on the other hand, is a homothallic fungus capable of self-fertilization, outcrossing, and vegetative reproduction (Vleugels et al. 2012). It is likely that selffertilization or vegetative reproduction are the primary means of reproduction in S. sclerotiorum, because outcrossing would require close proximity of genetically different isolates. The differences in reproductive mechanisms between species of Sclerotinia likely explain the intraspecies variation documented within the Polish $S$. trifoliorum isolates used in our study and the lack of intraspecies variation observed within the rDNA with the $S$. sclerotiorum isolates.

The dendrogram based on rDNA ITS sequences demonstrates that S. sclerotiorum and S. trifoliorum are closely related species, with both species grouping into a single clade. The dendrogram further demonstrates the lack of intraspecies variability within the rDNA of the $S$. sclerotiorum isolates used in our study and the presence of intraspecies variation with $S$. trifoliorum. Isolates of S. sclerotiorum from at least nine different host plants from the United States and Poland were examined and all isolates tested aggregated into one large group. Isolates of $S$. trifoliorum aggregated into a large subclade comprising three distinct groups, corresponding to the SNP at locations 120/376 in the ITS rDNA region sequenced. The results of our work and that of previous studies demonstrate the intraspecies variation within $S$. trifoliorum. Additional studies that include isolates from various geographic locations and additional host plants are required to fully exploit the utility of ITS rDNA for molecular characterization of $S$. trifoliorum.

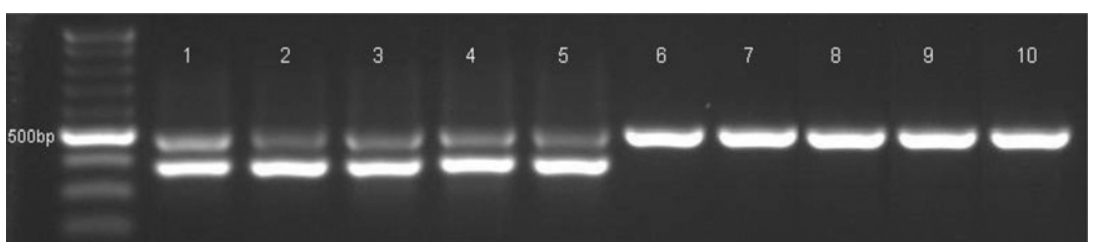

Fig. 2. Products of polymerase chain reaction amplification of Sclerotinia trifoliorum and S. sclerotiorum isolates using the primers TU1, TU2, and TU3. Products of 494 and $390 \mathrm{bp}$ in size were identified in S. trifoliorum isolates St 0211 TA, St 0511 TA, St 2113 TRe, St 01 TP, and St 09 TP (lanes 1 to 5, respectively). A single product of 494 bp was identified in S. sclerotiorum isolates Ss0311 BN, Ss 1212 HA, Ss5314 DC, Ss 12 GM, and Ss 39 GM (lanes 6 to 10, respectively).

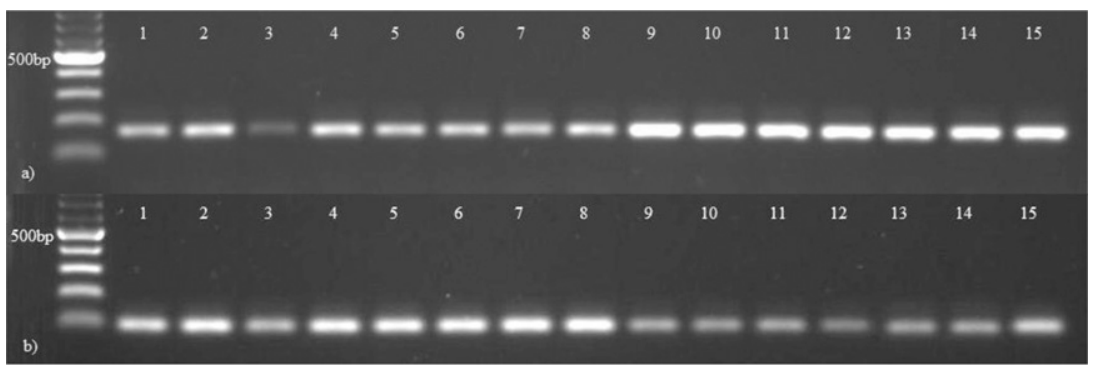

Fig. 3. Products of polymerase chain reaction amplification of Sclerotinia trifoliorum and S. sclerotiorum isolates originating from Poland using (row a) primers SSasprF and SSasprR and (row b) STCadF and STCadR. Products of appropriate size were identified using both primer sets for S. trifoliorum isolates St 0211 TA, St 0511 TA, St 1813 TP, St 2113 TRe, St 2413 TA, St 2813 TR, St 3313 TR, and CBS:122377 (lanes 1 to 8, respectively) and S. sclerotiorum isolates Ss 0311 BN, Ss 1212 HA, Ss 3813 BN, Ss 4812 AG, Ss 5112 PC, Ss 5213 LS, and Ss 5314 DC (lanes 9 to 15, respectively).

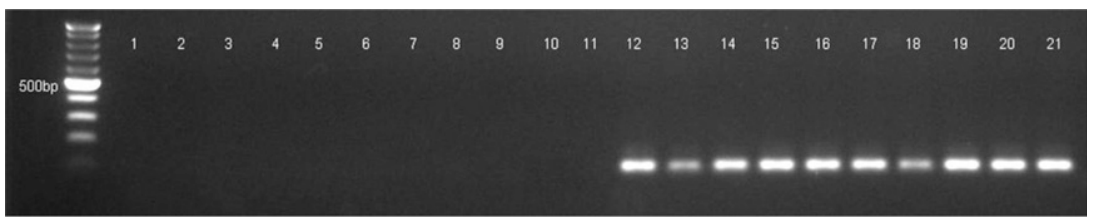

Fig. 4. Products of polymerase chain reaction amplification of Sclerotinia trifoliorum and S. sclerotiorum isolates using the primers SscadF1 and SscadR1. A product of 100 bp in size was identified only in S. sclerotiorum isolates Ss0311 BN, Ss 1212 HA, Ss 3813 BN, Ss 4812 AG, Ss 5112 PC, Ss 5213 LS, Ss5314 DC, Ss 12 GM, Ss 37 PV, and Ss 39 GM (lanes 12 to 21, respectively) whereas no PCR product was identified in S. trifoliorum isolates St 0211 TA, St 0511 TA, St 1813 TP, St 2113 TRe, St 2413 TA, St 2813 TR, St 3313 TR, St 01 TP, St 03 TP, St 09 TP, and CBS:122377 (lanes 1 to 11, respectively). 


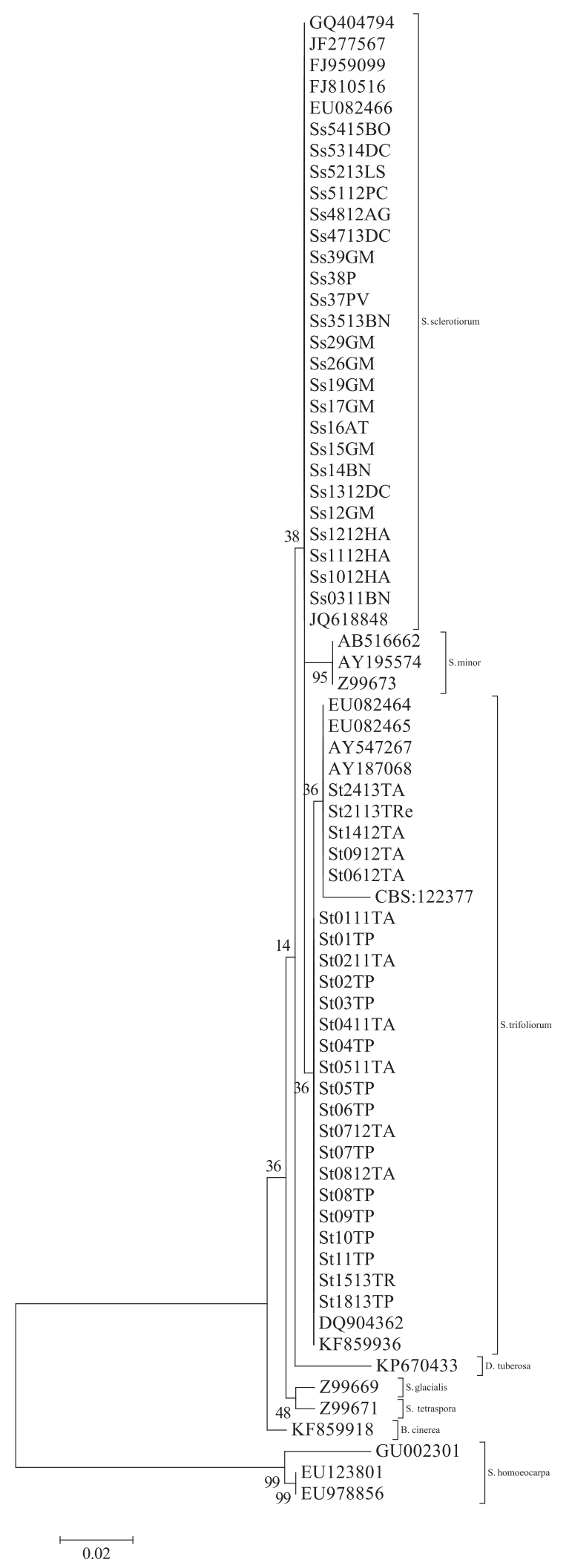

Fig. 5. Dendrogram constructed based on DNA sequences obtained from fragments of the ITS rDNA (amplicons from primers ITS1 and ITS4) from a subset of isolates (25 Sclerotinia trifoliorum and $23 \mathrm{~S}$. sclerotiorum isolates) using the unweighted pair group method with arithmetic mean algorithm. Ribosomal DNA sequences obtained from isolates examined were analyzed and compared with reference sequences obtained from the National Center for Biotechnology Information for six S. trifoliorum and six S. sclerotiorum isolates. Additional sequences from $S$. glacialis, S. homoeocarpa, S. minor, S. tetraspora, Dumontinia tuberosa, and Botrytis cinerea were included for additional comparison.
Amplification of Sclerotinia DNA with primers STcadF/STcadR and SSasprF/SSasprR was reported to be species specific in simplex and multiplex PCR (Abd-Elmagid et al. 2013). We found that amplification with both primer sets resulted in amplicons for all isolates tested, regardless of species, although the intensity of the band differed based on the specificity of the primer pair. A more intense band was obtained with isolates of S. sclerotiorum and primers SSasprF/ SSasprR than was obtained with isolates of S. trifoliorum. Similarly, a more intense band was obtained with isolates of S. trifoliorum and primers STcadF/STcadR than was obtained with isolates of $S$. sclerotiorum. Many factors can affect the intensity of bands obtained from PCR amplification; thus, basing species identification on band intensities is not possible. The presence of multiple amplicons, even of varying intensities, would confound the interpretation of multiplex PCR.

Primers designed to amplify the $\beta$-tubulin gene were effective in differentiating $S$. sclerotiorum and $S$. trifoliorum in this study. In addition, the primer pair developed for this study, SScadF1/SScadR1, amplified a single amplicon with $S$. sclerotiorum isolates but did not yield an amplification product with $S$. trifoliorum isolates. Based on the work presented here, PCR targeting the $\beta$-tubulin and calmodulin genes of the genus Sclerotinia is useful for rapid species identification. PCR analysis with the $\beta$-tubulin and calmodulin primer pairs is a relatively quick procedure utilizing supplies and equipment readily available to most laboratories. No extra step of DNA sequencing is necessary to differentiate between Sclerotinia spp. These PCR tools will be useful for differentiating between Sclerotinia spp. infecting clover and other plant hosts.

Finally, the genetic variation observed among isolates of $S$. trifoliorum is especially useful information for clover breeders and pathologists looking to develop resistant clover populations. The lack of knowledge about the genetic diversity of $S$. trifoliorum has been a hindrance to clover breeding programs in Europe (Vleugels et al. 2012). The information presented from our research suggests that, during selection of resistant lines, plants should be challenged by an array of $S$. trifoliorum isolates, not just one putatively aggressive isolate. The variation among these isolates may result in a range of aggressiveness levels or a differential pathogenicity signature. This was evident when Kura clover cultivars were sent from the United States to Poland. These cultivars were considered resistant to S. trifoliorum in the United States but were nearly decimated when planted in Poland.

\section{Acknowledgments}

A. Baturo-Ciesniewska acknowledges the receipt of a fellowship from the OECD Co-operative Research Programme: Biological Resource Management for Sustainable Agricultural Systems in 2014. We thank M. Kabbage for reviewing this manuscript. We thank C. Bradley, M. Chilvers, and D. Malvick for the field S. sclerotiorum isolate collections.

\section{Literature Cited}

Abd-Elmagid, A., Garrido, P. A., Hunger, R., Lyles, J. L., Mansfield, M. A. Gugino, B. K., Smith, D. L., Melouk, H. A., and Garzon, C. D. 2013. Discriminatory simplex and multiplex PCR for four species of the genus Sclerotinia. J. Microbiol. Methods 92:293-300.

Andrew, M., and Kohn, L. M. 2009. Single nucleotide polymorphism-based diagnostic system for crop-associated Sclerotinia species. Appl. Environ. Microbiol. 75:5600-5606.

Andrzejewska, J., Albrecht, K. A., and Harasimowicz-Hermann, G. 2014. Intercropping winter cereals with Caucasian clover for forage in northern Europe. Acta Agric. Scand. Sect. B Soil Plant Sci. 64:734-740.

Andrzejewska, J., Contreras-Govea, F. E., Pastuszka, A., and Albrecht, K.A. 2016. Performance of Kura clover compared to that of perennial forage legumes traditionally cultivated in central Europe. Acta Agric. Scand. Sect. B Soil Plant Sci. 66:516-522.

Barnett, O. W., and Diachun, L. A. 1985. Virus diseases of clovers. Pages 235-268 in: Clover Science and Technology. Agron. Monogr. 25. N. L. Taylor, ed. ASACSSA-SSSA, Madison, WI

Barnett, O. W., and Gibson, P. B. 1975. Identification and prevalence of white clover viruses and the resistance of Trifolium species to these viruses. Crop Sci. 15:32-37.

Baturo-Ciesniewska, A., Andrzejewska, J., Albrecht, K., Sadowski, Cz., and Lenc, L. 2013. First report of Sclerotinia stem blight caused by Sclerotinia trifoliorum on Trifolium ambiguum in Poland. Plant Dis. 97:142.

Bryant, W. G. 1974. Caucasian clover (Trifolium ambiguum Bieb.): A review. J. Aust. Inst. Agric. Sci. 40:11-19. 
Carbone, I., and Kohn, L. M. 1993. Ribosomal DNA sequence divergence within internal transcribed spacer 1 of the Sclerotiniaceae. Mycologia 85:415-427.

Freeman, J., Ward, E., Calderon, C., and McCartney, A. 2002. A polymerase chain reaction (PCR) assay for the detection of inoculum of Sclerotinia sclerotiorum. Eur. J. Plant Pathol. 108:877-886.

Harasimowicz-Hermann, G., and Andrzejewska, J. 1999. Trifolium ambiguumCharakterystyka gatunku i możliwości introdukcji w Polsce. Zesz. Nauk. Akad. Roln. im. H. Kollataja Krakowie Roln. 347:113-121.

Holst-Jensen, A., Vaage, M., Schumacher, T., and Johansen, S. 1999. Structural characteristics and possible horizontal transfer of group I introns between closely related plant pathogenic fungi. Mol. Biol. Evol. 16:114-126.

Kammes, K. L., Heemink, G. B., Albrecht, K. A., and Combs, D. K. 2008. Utilization of Kura clover-reed canarygrass silage versus alfalfa silage by lactating dairy cows. J. Dairy Sci. 91:3138-3144.

Kannenberg, L. W., and Elliott, F. C. 1962. Ploidy in Trifolium ambiguum M. Bieb. in relation to some morphological and physiological characters. Crop Sci. 2:378-381.

Kohn, L. M. 1979. Delimitation of the economically important plant pathogenic Sclerotinia species. Phytopathology 69:881-886.

Kohn, L. M., Petsche, D. M., Bailey, S. R., Novak, L. A., and Anderson, J. B. 1988. Restriction fragment length polymorphisms in nuclear and mitochondrial DNA of Sclerotinia species. Phytopathology 78:1047-1051.

Marum, P., Smith, R. R., and Grau, C. R. 1994. Development of procedures to identify red clover resistant to Sclerotinia trifoliorum. Euphytica 77:257-261.

Mouriño, F., Albrecht, K. A., Schaefer, D. M., and Berzaghi, P. 2003. Steer performance on Kura clover-grass and red clover-grass mixed pastures. Agron. J. 95:652-659.

Njambere, E. N., Chen, W., Frate, C., Wu, B.-M., Temple, S. R., and Muehlbauer, F. J. 2008. Stem and crown rot of chickpea in California caused by Sclerotinia trifoliorum. Plant Dis. 92:917-922.

Patrick, H. N., Lowther, W. L., and Trainor, K. D. 1994. Inoculation for successful establishment of Caucasian clover. Proc. N. Z. Grassl. Assoc. 56:101-105.

Pederson, G. A., and McLaughlin, M. R. 1989. Resistance to viruses in Trifolium interspecific hybrids related to white clover. Plant Dis. 73:997-999.

Pederson, G. A., and Windham, G. L. 1989. Resistance to Meloidogyne incognita in Trifolium interspecific hybrids and species related to white clover. Plant Dis. 73:567-589.

Peterson, P. R., Sheaffer, C. C., Jordan, R. M., and Christians, C. J. 1994. Responses of Kura clover to sheep grazing and clipping: I. Yield and forage quality. Agron. J. 86:655-660.

Powers, K. S., Steadman, J. R., Higgins, B. S., and Powers, T. O. 2001. Intraspecific variation within North American Sclerotinia trifoliorum isolates characterized by nuclear small subunit rDNA introns. Pages 95-96 in: Proc. Sclerotinia 2001-XI Int. Sclerotinia Workshop, York, England. C. S. Young and K. J. D. Hughes, eds. Central Science Laboratory, York, England.

Pratt, R. G., and Rowe, D. E. 1995. Comparative pathogenicity of isolates of Sclerotinia trifoliorum and Sclerotinia sclerotiorum on alfalfa cultivars. Plant Dis. 79:474-477.

Pryor, H. N., Lowther, W. L., and Trainor, K. D. 1996. Natural reseeding of Caucasian clover (Trifolium ambiguum) in tussock grasslands. Proc. N. Z Grassl. Assoc. 58:171-175.

Saharan, G. S., and Mehta, N. 2008. Sclerotinia Diseases of Crop Plants: Biology, Ecology and Disease Management. Springer, New Delhi.

Sheaffer, C. C., and Marten, G. C. 1991. Kura clover forage yield, forage quality, and stand dynamics. Can. J. Plant Sci. 71:1169-1172.

Speer, G. S., and Allinson, D. W. 1985. Kura clover (Trifolium ambiguum): Legume for forage and soil conservation. Econ. Bot. 39:165-176.

Steiner, J. J., and Snelling, J. P. 1994. Kura clover seed production when intercropped with wheat. Crop Sci. 34:1330-1335.

Tamura, K., Stecher, G., Peterson, D., Filipski, A., and Kumar, S. 2013. MEGA6: Molecular Evolutionary Genetics Analysis Version 6.0. Mol. Biol. Evol. 30: 2725-2729.

Tariq, V. N., Gutteridge, C. S., and Jeffries, P. 1985. Comparative studies of cultural and biochemical characteristics used for distinguishing species within Sclerotinia. Trans. Br. Mycol. Soc. 84:381-397.

Taylor, N. L. 2008. A century of clover breeding developments in the United States. Crop Sci. 48:1-13.

Taylor, N. L., and Smith, R. R. 1997. Kura clover (Trifolium ambiguum M.B.) breeding, culture and utilization. Adv. Agron. 63:153-178.

Vleugels, T., Baert, J., and van Bockstaele, E. 2013. Morphological and pathogenic characterization of genetically diverse Sclerotinia isolates from European red clover crops (Trifolium pratense L.). J. Phytopathol. 161:254-262.

Vleugels, T., De Riek, J., Heungens, K., van Bockstaele, E., and Baert, J. 2012. Genetic diversity and population structure of Sclerotinia species from European red clover crops. J. Plant Pathol. 94:493-503.

White, T. J., Bruns, T., Lee, S., and Taylor, J. 1990. Amplification and direct sequencing of fungal ribosomal RNA genes for phylogenetics. Pages 315-322 in: PCR Protocols: A Guide to Methods and Applications. M. A. Innis, D. H. Gelfand, J. J. Sninsky, and T. J. White, eds. Academic Press, San Diego, CA.

Yli-Mattila, T., Kalko, G., Hannukkala, A., Paavanen-Huhtala, S., and Hakala, K. 2010. Prevalence, species composition, genetic variation and pathogenicity of clover rot (Sclerotinia trifoliorum) and Fusarium spp. in red clover in Finland. Eur. J. Plant Pathol. 126:13-27.

Zemenchik, R. A., Albrecht, K. A., Boerboom, C. M., and Lauer, J. G. 2000. Corn production with Kura clover as a living mulch. Agron. J. 92:698-705. 